\title{
Discapacidad intelectual, evolución social del concepto
}

Intellectual Disability, social evolution of the concept
Deficiência Intelectual, a evolução social do conceito
Fecha de Recepción

06 de agosto de 2014
Aceptado para su publicación

II de noviembre de 2014
Sofía de los Milagros Alí Prof. Adjunta Cátedra Odontología Social y Comunitaria

Roxana Lilian Blanco Auxiliar de Primera Categoría Cátedra Odontología Social y Comunitaria Facultad de Odontología - UNNE

\section{Resumen}

El presente artículo, tiene como finalidad partir de la concepción que la discapacidad, es una construcción social, así mismo se desarrolla la evolución del concepto social. Dicha evolución lleva consigo un cambio en el lenguaje, que es reflejo de la sociedad del momento y revela las concepciones. Es así que las personas con discapacidad reciben diversas denominaciones. $\mathrm{Si}$ bien, la sociedad está cada vez más informada sobre la discapacidad no se debe olvidar que todavía existe un lenguaje incorrecto en la misma.La relevancia de realizar una mirada crítica al empleo de estos conceptos, en este análisis, radica asimismo en que se considera que la forma en que la sociedad se refiere a este colectivo, habla de cómo se relaciona con el mismo, considerándolos o ubicándolos en el nivel de "los otros".

\section{Palabras claves}

Discapacidad. OMS. Sociedad. Persona.

\section{Summary}

This article aims from the conception that disability is a social construction, is the change of the social concept is developed. This evolution involves a change in language, that reflects society at the time and reveals the conceptions. So that people with disabilities receive different names. While society is increasingly informed about disabilities must not be forgotten that 
there is still a bad language in it. The importance of taking a critical look at the use of these concepts, this analysis also is that it is considered that the way in which the society refers to this group, speaks of how it relates to the same, considering them or placing them in the level of "others".

\section{Keywords \\ Disability. WHO. Society. Person.}

\section{Resumo}

Este artigo tem como objetivo a partir da concepção de que a deficiência é uma construção social, é a alteração do conceito social é desenvolvido. Esta evolução implica uma mudança na linguagem, que reflectem a sociedade na época e revela as concepções. Para que as pessoas com deficiência recebem diferentes denominações. Enquanto a sociedade está cada vez mais informados sobre deficiência não se deve esquecer que ainda há uma má língua nele. A importância de se ter um olhar crítico sobre o uso desses conceitos, esta análise também é que se considera que a forma como a sociedade se refere a este grupo, fala de como ele se relaciona com o mesmo, considerando-los ou colocá-los no nível de "outros".

\section{Palavras chave \\ Deficiência. OMS. Society. Pessoa.}

\section{Desarrollo}

La evolución del concepto social de la discapacidad intelectual, se debe enmarcar dentro de la evolución del concepto de la discapacidad en general. Comenzaremos partiendo por exponer un concepto de discapacidad y su evolución. Se parte de las clasificaciones internacionales propuestas por la Organización Mundial de la Salud (OMS)': la clasificación Internacional de Deficiencias, Discapacidades y Minusvalías (CIDDM) ${ }^{2}$ de 1980, que surge de trabajos iniciados en 1972 a partir de la Clasificación Internacional de Enfermedades (CIE),ya que ésta resultaba insuficiente para explicar y clasificarlas consecuencias de la enfermedad sobre el desarrollo global de la persona; y la Clasificación Internacional del Fun- cionamiento, la Discapacidad y la Salud $(\mathrm{CIF})^{3}$, del 200I, que constituye la segunda versión de la CIDDM.

En estos dos modelos opuestos está basada la CIF. Con el fin de conseguir la integración delas diferentes dimensiones del funcionamiento, la clasificación utiliza un enfoque "biopsicosocial". Por lo tanto, la CIF intenta conseguir una síntesis y con ella, proporcionar una visión coherente de las diferentes dimensiones de la salud desde una perspectiva biológica, individual y social ${ }^{4}$.

Se han propuesto diferentes modelos conceptuales para explicar y entender la discapacidad $y$ el funcionamiento, que pueden resumirse en la dialéctica entre "modelo médico" y "modelo social" . El modelo médico considera la discapacidad como un problema de la persona directamente causado por una enfermedad, trauma o condición de salud, que requiere de cuidados médicos prestados en forma de tratamiento individual por profesionales, encaminado a conseguirla cura, o una mejor adaptación de la persona y un cambio de su conducta. El modelo social de la discapacidad, considera el fenómeno fundamentalmente como un problema de origen social y principalmente como un asunto centrado en la completa integración de las personas en la sociedad. La discapacidad no es un atributo de la persona, sino un complicado conjunto de condiciones, muchas de las cuales son creadas por el contexto/entorno social. Teniendo en cuenta lo expuesto, se puede decir que:

"La discapacidad está definida como el resultado de una compleja relación entre la condición de salud de una persona y sus factores personales, y los factores externos que representan las circunstancias en las que vive esa persona.

El constructo actual de discapacidad se centra en la expresión de limitaciones en el funcionamiento individual en un contexto social y que representa una desventaja substancial para el individuo. La discapacidad tiene su origen en una condición de salud que da lugar a déficit en el cuerpo y las estructuras, limitaciones en la actividad y restricciones en la participación en el contexto de los factores personales y ambientales $^{6}$. Antes se hablaba de retraso mental, minusvalía psíquica, discapacidad del aprendizaje. Actualmente se tiende a considerar como políticamente correcto el termino discapacidad 
REVISTA FACULTAD DE ODONTOLOGÍA

ISSN No 1668-7280 - Vol. VIII No 1 - 2015

intelectual. Sin embargo, el constante cambio de nombre no obedece a criterios terminológicos sino al estigma asociado al retraso mental. Como este se asocia al problema y no a la denominación del mismo, los distintos términos finalizan absorbidos por dicho estigma, lo cual se hizo necesario cambiarlos.

La terminología utilizada para denominar lo que ahora se llama discapacidad intelectual ha cambiado históricamente, se han utilizado cuatro enfoques generales (criterio social, clínico, intelectual y dual) con propósitos de definición y clasificación. Los vestigios de estos cuatro enfoques son aún evidentes en las discusiones actuales relacionadas con quién es (o debería ser) diagnosticado como un individuo con discapacidad intelectual ${ }^{7}$.

Luckasson y Reeve $(200 \mathrm{I})^{8}$ señalaron cinco factores importantes que se han de tener en cuenta cuando se elige un término. Primero, el término debería ser específico, referirse a una entidad única, permitir la diferenciación de otras entidades y mejorar la comunicación. Segundo, los distintos grupos implicados (individuos, familias, escuelas, clínicos, abogados, médicos, profesionales de las organizaciones, investigadores y políticos) deben utilizarlo de forma consistente. Tercero, el término debe representar de forma adecuada el conocimiento actual y poder incorporar el conocimiento nuevo según ocurren los avances científicos. Cuarto, debería ser lo suficientemente robusto en su operacionalización para permitir su uso con múltiples propósitos, incluyendo definir, diagnosticar, clasificar y planificar los apoyos. Quinto, debería reflejar un componente esencial del hecho de poner nombre a un grupo de personas, que es comunicar valores importantes, especialmente hacia el grupo.

Definir hace referencia a explicar de forma precisa el término y establecer el significado y los límites del término. La definición oficial de discapacidad intelectual/retraso mental es la de la AAIDD (American Association on Intellectual and Developmental Disabilities) (anteriormente la AAMR). La definición del Manual de la AAMR ('American Association on Mental Retardation')' del 2002 permanece en vigor ahora y para el futuro próximo. Esta definición que se presenta aquí en cursiva es la que sustituye el término retraso mental por discapacidad intelectual:

"La Discapacidad Intelectual se caracteriza por limitaciones significativas en el funcionamiento intelectual y en la conducta adaptativa, expresada en las habilidades adaptativas conceptuales, sociales y prácticas. La discapacidad se origina antes de los 18 años". Esto implica una limitación en las habilidades que la persona aprende para funcionar en su vida diaria y que le permiten responder en distintas situaciones y en lugares (contextos) diferentes. Dicha discapacidad se expresa cuando una persona con limitaciones significativas interactúa con el entorno. Es decir, que obedece tanto de la propia persona como de las barreras u obstáculos que tiene el mismo. Esto dependiendo si es un entorno más o menos facilitador, la discapacidad se expresará de manera diferente. La nueva terminología, empleada es discapacidad intelectual, y se elimina definitivamente la expresión retraso mental. Se actualiza el modelo conceptual desde una perspectiva socio-ecológica, multifactorial de la etiología y la distinción entre una definición operativa y otra constitutiva de la condición ${ }^{10}$.

\section{Modelo socio-ecológico}

El modelo socio ecológico de la discapacidad intelectual es importante para la comprensión de la condición y el enfoque que tomamos porque explica la misma en términos de: a) expresión de limitaciones en el funcionamiento individual dentro de un contexto social; b) visión de las personas con un origen en factores orgánicos y/o sociales; $y c$ ) entendimiento que estos factores orgánicos y sociales causan limitaciones funcionales.

\section{Explicación multifactorial de la etiología de la discapacidad intelectual:}

Históricamente, se distinguía entre causas orgánicas y ambientales de la condición a las que se hacía referencia como deficiencia mental, retraso mental o [más recientemente] discapacidad intelectual.

Con la introducción en 1992 de la $9^{a}$ edición del Manual de la AAMR se inició un enfoque multifactorial para explicar la etiología de la condición.

Una definición constitutiva: 
Una definición constitutiva de discapacidad intelectual define la condición en términos de limitaciones en el funcionamiento humano, implica una comprensión de la discapacidad consistente con una perspectiva multidimensional y socio ecológica, y subraya el rol significativo que los apoyos individualizados desempeñan en la mejora del funcionamiento humano.

Este tipo de discapacidad tiene un impacto importante en la vida de la persona y de su familia, dado que generalmente es permanente.

Dentro de este tipo de discapacidad se encuentran las siguientes deficiencias:

$\checkmark$ Síndrome de Down

$\checkmark$ Síndrome de Williams

$\checkmark$ Síndrome de Noonan

$\checkmark$ Síndrome de Cornelia de Lange (SCdL)

$\checkmark$ Autismo

$\checkmark$ Síndrome de Asperger (SA)

No debemos considerar a la discapacidad como una enfermedad, debemos ver a estos individuos:

$\checkmark$ Como persona, como un ciudadano más.

$\checkmark$ A cada persona en su entorno, con sus capacidades y sus limitaciones.

$\checkmark \quad$ Con posibilidades de progresar si le damos los apoyos adecuados.

\section{Conclusión}

La principal razón por la cual se sugirió un cambio en la terminología deriva del carácter peyorativo del significado de retraso mental que, además, reduce la comprensión de las personas con limitaciones intelectuales a una categoría diagnóstica nacida desde perspectivas psicopatológicas. La discapacidad intelectual debe concebirse hoy desde un enfoque que subraye en primer lugar a la persona como a cualquier otro individuo de nuestra sociedad. En conclusión, estos cambios que se están produciendo internacionalmente en el campo de la discapacidad no sólo están modificando el modo en que vemos a las personas con discapacidad, sino también nuestra terminología. Además, el tener una nueva forma de pensar, amplía nuestro enfoque respecto a las personas con discapacidad.

\section{Bibliografía}

I. OMS (200I). Clasificación Internacional del Funcionamiento, de la Discapacidad y de la Salud. OMS, OPS, IMSERSO, Madrid, España.

2. Organización Mundial de la Salud, "Clasificación Internacional de Deficiencias, Discapacidades y Minusvalías", IMSERSO, edición 1997. Madrid.

3. Querejeta Miguel. Las herramientas del nuevo paradigma de la salud en el siglo XXI: CIE / CIF. Rev. Esp. Salud Pública [revista en la Internet]. 2009 Dic [citado 2014 Septiembre 25] ; 83(6): 77I-773.

4. Fernández-López Juan Antonio, FernándezFidalgo María, Geoffrey Reed, Stucki Gerold, Cieza Alarcos. Funcionamiento y discapacidad: la clasificación internacional del funcionamiento (CIF). Rev. Esp. Salud Pública [revista en la Internet]. 2009 Dic [citado 2014 Jul I6] ; 83(6): 775-783.

5. Cáceres Rodríguez, Celsa. Sobre el concepto de discapacidad. Una revisión de las propuestas de la OMS. Revista Electrónica de Audiología Vol. 2 (c) Auditio.com 2004. Disponible en: http://www. auditio.com/revista/pdf/vol2/3/020304.pdf

6. Schalock RL, et al. Intellectual disabilities: definitions, classification, and system of support. II th ed. Washington, American Association on Intellectual and Developmental Disabilities, 2010.

7. Switzky, H.N. y Greenspan, S. (2006a). What is mental retardation: Ideas for an evolving disability. Washington, DC: American Association on Mental Retardation.

8. Schalock, Robert L., Luckasson, Ruth A., and Shogren, Karrie A. The Renaming of Mental Retardation: Understanding the Change to the Term Intellectual Disability. Intellectual and Developmental Disabilities: April 2007, Vol. 45, No. 2, pp. II6-124.

9. Luckasson, R., Borthwick-Duffy, S., Buntix, W.H.E., Coulter, D.L., Craig, E.M., Reeve, A., y cols. (2002). Mental Retardation. Definition, classification and systems of supports (10th ed.). Washington, DC: American Associationon Mental Retardation. [Traducción al castellano de M.A. Verdugo y C. Jenaro (en prensa). Madrid: Alianza Editorial].

10. Verdugo Alonso, Miguel Ángel, Schalock, Robert L. Últimos avances en el enfoque y concepción de las personas con discapacidad intelectual. Revista Española sobre Discapacidad Intelectual Vol 4I (4), Núm. 236, 2010 Pág. 7 a pág. 2 I 